\title{
NOMENCLATURA Y USOS DE LOS PATOS COMERCIALIZADOS EN MERCADOS DE LA PROVINCIA DE PICHINCHA, ECUADOR
}

\section{NOMENCLATURE AND USES OF DUCKS COMMERCIALIZED IN MARKETS FROM PICHINCHA PROVINCE, ECUADOR}

Henry Paul MONAR-BARRAGÁN, Camilo Andrés ROSERO-GÓMEZ, Stephanie Carolina LÓPEZ TIPÁN, Iván Vinicio JÁCOME-NEGRETE

Facultad de Ciencias Biológicas de la Universidad Central del Ecuador, calle Iquique N14-121 y Sodiro-Itchimbía, EC170136, Quito, Ecuador; e-mail: hpmonarb@gmail.com

\section{RESUMEN}

Sublted: 25/05/2020; Accepted: $19 / 06 / 2020$

En siete mercados de la provincia de Pichincha se recopiló información sobre la nomenclatura y usos de los patos (familia Anatidae) comercializados localmente, con el fin de incrementar el conocimiento etno-ornitológico actual sobre este grupo en Ecuador. Se realizaron 45 entrevistas semiestructuradas a comerciantes y compradores de los mercados. La edad promedio de los participantes fue de 56 años, con un rango de edad entre los 25 a 83 años. Fueron identificadas tres especies: el pato mostachón, nacional, runa o criollo Cairina moschata (Linnaeus, 1758); el pato Pekín, chino o blanco Anas platyrhynchos domesticus Linnaeus, 1758; y el ganso o pato ganso Anser anser (Linnaeus, 1758). Las tres especies son usadas principalmente dentro de las categorías de alimentación (68\%) y crianza $(22 \%)$. Dentro de alimentación se incluyen preparaciones nacionales e internacionales además del consumo de huevos. Además, las dos especies de patos registran usos en las categorías medicinal (4\%), didáctica (1\%) y tenencia de mascotas (11\%). En la categoría medicinal se menciona que con el consumo de huevos se reducen los malestares de la menopausia y afecciones de garganta. El comercio de los individuos se realiza en diferentes estadios de edad: polluelo (15-30 días de edad), juvenil ( 3 a 5 meses de nacido), y adulto (individuos de 7 meses en adelante). Los juveniles son más comercializados ya que pueden emplearse de inmediato como fuente de alimento o para reproducción. La especie nativa es preferida en el comercio, debido a las facilidades de cuidado y mejores características reproductivas, de acuerdo a los participantes.

Palabras-clave: Etno-ornitología, gansos, patos, usos.

\begin{abstract}
In seven markets from Pichincha province, information was collected about nomenclature and uses of ducks (Anatidae family) commercialized locally, in order to increase current ethno-ornithological knowledge about this group in Ecuador. 45 semi-structured interviews were conducted with merchants and buyers in the markets. The average age of participants was 56 years, with an age range between 25 to 83 years. Three species were identified: the mustard, national, runa or Creole duck Cairina moschata (Linnaeus, 1758), the Peking, Chinese or white duck Anas platyrhynchos domesticus Linnaeus, 1758, and, the goose or goose duck Anser anser (Linnaeus, 1758). The three species are mainly used within the feeding (68\%) and breeding (22\%) categories. Food includes national and international preparations, and the consumption of eggs. In addition, the two species of ducks register uses in the medicinal (4\%), didactic (1\%) and family pet $(11 \%)$ categories. In the medicinal category, it is mentioned that the consumption of eggs reduces the discomforts of menopause and throat conditions. Trading of individuals is carried out at different age stages: chick (15-30 days old), juvenile ( 3 to 5 months old), and adult (individuals 7 months and older). Juveniles are more commercialized because they can be used immediately as a food source or for reproduction. The native species is preferred for trade, due to the care facilities and better reproductive characteristics, according to the participants.
\end{abstract}

Keywords: Ethno-ornithology, geese, ducks, uses. 


\section{INTRODUCCIÓN}

La familia Anatidae incluye aves como los patos, cisnes y gansos. Estas aves presentan un pico plano y redondeado con laminillas córneas en sus bordes laterales y patas palmeadas por sus hábitos acuáticos (ALBUJA et al., 2012). Según Freile y Restall (2018), en Ecuador se han registrado 20 especies de patos silvestres y un ganso.

El pato mostachón Cairina moschata (Linnaeus, 1758) es una especie nativa que ha sido el principal objeto de un proceso de domesticación local en Ecuador. Estudios arqueológicos reportan diversas evidencias del conocimiento y uso de los patos por culturas precolombinas. Existen representaciones del pato en cerámica, con una antigüedad de aproximadamente 2000 años, correspondientes a los períodos Formativo Tardío y de Integración (MINISTERIO DE CULTURA Y PATRIMONIO, 2016).

Gutiérrez (2009) menciona evidencias claves que describen vínculos culturales con los patos locales desde los años 1000 a.C. en la costa ecuatoriana. El autor reporta la existencia de vasijas con forma de Anseriformes (posiblemente del pato María Dendrocygna bicolor Vieillot, 1816) hechas por la cultura Engoroy (1000 al 300 a.C.) en el período formativo final; de igual forma, indica que en Salango (100 a.C. a 800 d.C.) se encontraron restos del pato C. moschata como uno de los animales domesticados por la cultura Guangala. Así mismo menciona que varias culturas de la costa pertenecientes al período de Desarrollo Regional (355 a.C.) tales como Tumaco-Tolita, Tiaone, JamaCoaque, Bahía y Guangala fueron posiblemente las responsables de la dispersión del pato mostachón C. moschata, desde México a Perú, a través de sus viajes marítimos de intercambio y comercio de Spondylus. Finalmente, el autor también indica que en crónicas españolas que datan de la conquista, ya se reporta la presencia de patos domésticos $C$. moschata como una de las especies domesticadas por la cultura Manteña para el período de integración (500 a 1500 d.C.).

Existen también reportes de la época prehispánica para los Andes ecuatorianos que mencionan a $C$. moschata, llamado localmente xuta, como un ave buena para la alimentación (STAHL et al., 2006). Esta especie fue usada en la cocina de aquella época en Quito, en la que los patos criados en el lago de Iñaquito y las pozas de Turubamba, eran consumidos cocinados o asados por los habitantes de la ciudad (PAZOS-BARRERA, 2008).

Debido a su rápida reproducción, la crianza de los patos resulta más sencilla en comparación a las gallinas. Los patos no son exigentes en aspectos de comida o espacio; son más resistentes a enfermedades y condiciones ambientales adversas (AVILEZ y CAMIRUAGA, 2006; LLACSAHUACHE, 2019), lo que hace importante estudiar a este grupo de aves con fines comerciales.

El estudio de los aspectos culturales y biológicos de los usos que tienen los animales, es uno de los campos de actuación de la etnozoología (SANTOS FITA et al., 2009). El empleo del animal completo, parte o un derivado de este en la gastronomía local o como un producto zooterapeútico es un elemento importante del patrimonio zoocultural material (VARGAS-CLAVIJO, 2009). La zooterapia es el empleo de los animales o de productos de estos para el tratamiento de enfermedades humanas (NÓBREGA, 2009).

En este contexto, el objetivo del presente estudio fue recopilar información sobre la nomenclatura y los diferentes usos de las especies de patos que son habitualmente objeto de comercialización en varios mercados de la provincia de Pichincha. Con el fin de aportar al limitado conocimiento etno-ornitológico existente sobre estas aves en Ecuador y como un punto de partida que pueda fortalecer la conservación del conocimiento local asociado a su manejo.

\section{MATERIALES Y MÉTODOS}

\section{Área de Estudio}

El estudio se realizó en siete mercados de tres cantones en la provincia de Pichincha: el Mercado de Sangolquí del Cantón Rumiñahui, el Mercado de Machachi en el Cantón Mejía y los Mercados Las Cuadras, San Roque, Calderón, Chiriyacu y La Ofelia que se localizan en el Cantón Quito (CONSEJO PROVINCIAL DE PICHINCHA, 2002) (Figura 1). Todas las localidades pertenecen al piso zoogeográfico templado (ALBUJA et al., 2012); pese a estar ubicados en zonas urbanas de la provincia, los mercados constituyen un mosaico cultural y centros de comercio, donde se incluye la fauna urbana tanto nativa como introducida (CAZAMAJOR y MOYA, 1984).

La población de la provincia de Pichincha está principalmente representada por el grupo étnico mestizo y minorías de blancos e indígenas (TIGMASA-MENA, 2018). Se trabajó con los 
vendedores establecidos en los mercados, cuya actividad económica se relaciona al comercio de las especies de avifauna de granja. De igual forma, se entrevistó a los compradores de las aves sin considerar su proveniencia con el fin de indagar sobre la nomenclatura y el uso que le dan a las especies de patos. El rango de edad de los entrevistados estuvo entre 25 y 83 años, independientemente del género.

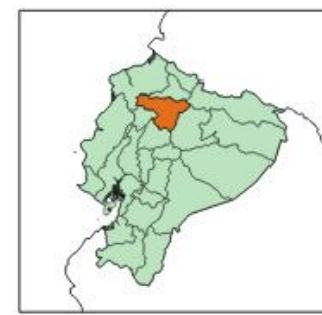

Leyenda

Mercados Visitados

Calderón

Chiriyaa

- La Ofelia

- Las Cuadras

- Machadi

- San Roque

- Sangolqui

Provinaas

$\square$ PICHINCHA

— Límites Políticos

Datum:WGS84

Fuente: Instituto Geogáfico Militor

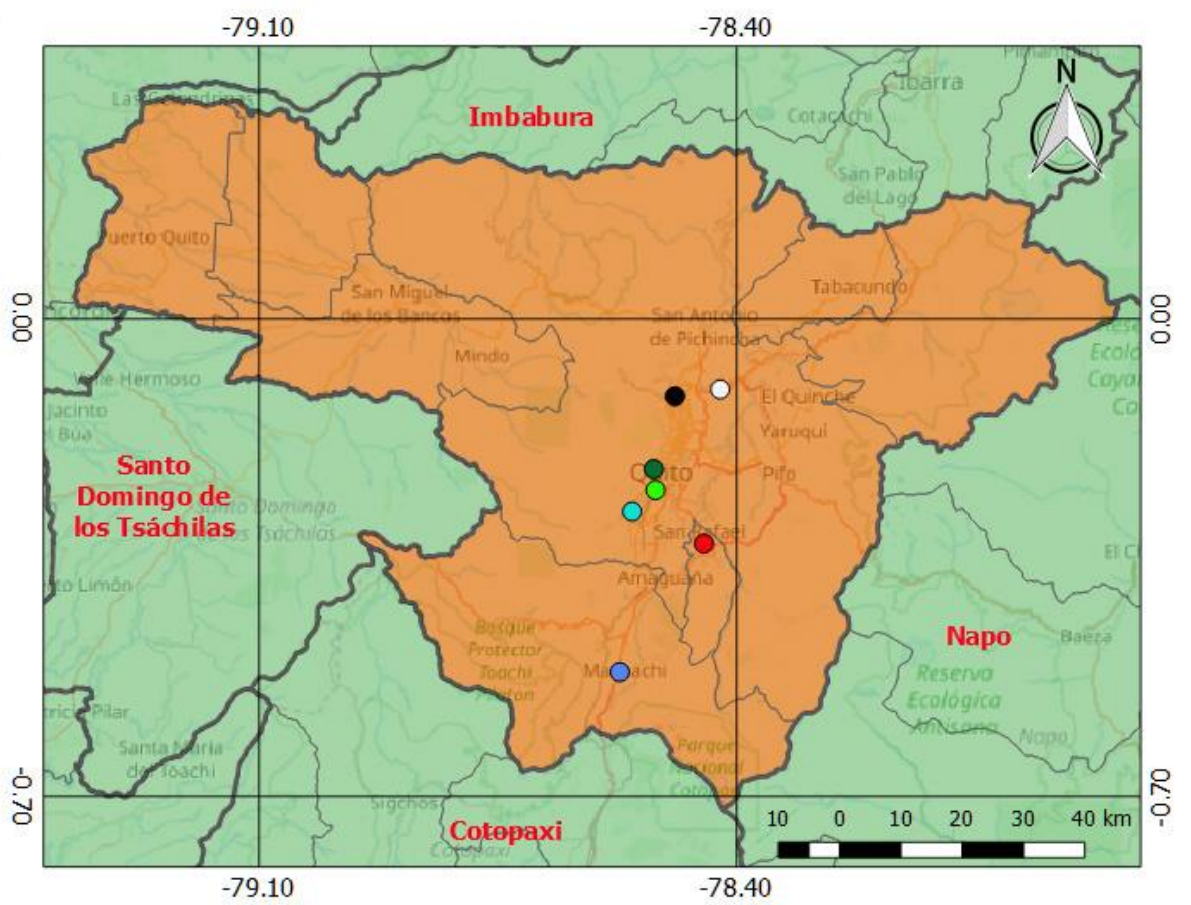

Figura 1: Ubicación de los Mercados donde se realizó el levantamiento de información, PichinchaEcuador.

\section{Métodos}

Para el levantamiento de datos en campo se utilizó la técnica de entrevistas semiestructuradas (VÁSQUEZ, 2014), fueron aplicadas a un total de 45 personas, compuestas por 33 comerciantes y 12 compradores en los siete mercados estudiados. El contenido de la entrevista aplicada incluyó dos secciones: la primera sección enfocada a documentar los aspectos demográficos de los entrevistados, y la segunda sección estructurada con preguntas sobre los nombres comunes de las aves comercializadas, los usos conocidos y otros aspectos de su comercialización tales como su origen, edad de los animales y frecuencia de compra/venta, en concordancia con el procedimiento propuesto por Bernard (1996). Las entrevistas se realizaron previo consentimiento informado de las personas participantes, en horas de la mañana durante los días de feria establecidos para cada mercado, de mayo a agosto de 2019.

Para la tabulación de los datos recopilados en el campo, así como su representación gráfica y empleo de medidas de estadística descriptiva, se utilizó el software de cálculo Microsoft Excel® 2013, conforme sugieren Gutiérrez-Cantuña y Guainilla-Maldonado (2018). Para la identificación taxonómica de las aves se utilizó la lista de aves de la Guía de aves del Ecuador de McMullan y Navarrete (2017). Los datos demográficos de los participantes fueron tabulados de manera independiente a los datos de nomenclatura y uso de las aves, debido a que una sola persona entrevistada brindó información sobre varias especies de patos.

\section{RESULTADOS \\ Datos Demográficos}

La edad promedio de las personas entrevistadas $(n=45)$ en los mercados fue de 56 años (Tabla 1). La edad máxima encontrada fue de 83 años, mientras que la mínima fue de 25 años. El $68.89 \%$ de los entrevistados $(n=31)$ están sobre los 50 años y, de estos, catorce personas son de la tercera edad.

Como se observa en la Tabla 1 y Figura 2, el grupo dominante de comerciantes de aves de acuerdo al sexo corresponde a las mujeres. El $29 \%$ de entrevistadas tienen como principal ocupación 
el de amas de casa y al tratarse de entrevistas en mercados, un alto porcentaje de personas (65\%) se auto identificaron como comerciantes.

Durante la investigación fue posible entrevistar a personas que indicaron distinto lugar de origen, incluyendo a un ciudadano extranjero (Figura 3). Si bien el lugar con mayor número de entrevistados corresponde a la Zona de Quito, la mayoría de las personas son originarias de sitios alejados al casco urbano, que no se corresponde necesariamente con la ubicación de los mercados donde fueron entrevistados.

Tabla 1: Datos demográficos de las personas entrevistadas.

\begin{tabular}{lccc}
\hline \multirow{2}{*}{ Mercado/Feria } & \multicolumn{2}{c}{ Sexo } & \multirow{2}{*}{ Edad Promedio (años) } \\
\cline { 2 - 3 } & Femenino & Masculino & 59 \\
\hline Calderón $(\mathrm{n}=5)$ & 7 & 0 & 57 \\
Chiriyacu $(\mathrm{n}=4)$ & 4 & 1 & 57 \\
La Ofelia $(\mathrm{n}=4)$ & 6 & 0 & 60 \\
Las Cuadras $(\mathrm{n}=6)$ & 6 & 0 & 58 \\
Machachi $(\mathrm{n}=4)$ & 3 & 1 & 45 \\
San Roque $(\mathrm{n}=8)$ & 4 & 4 & 59 \\
Sangolquí $(\mathrm{n}=9)$ & 9 & 0 & $\mathbf{5 6}$ \\
\multicolumn{1}{c}{ TOTAL } & $\mathbf{3 9}$ & $\mathbf{6}$ & \\
\hline
\end{tabular}

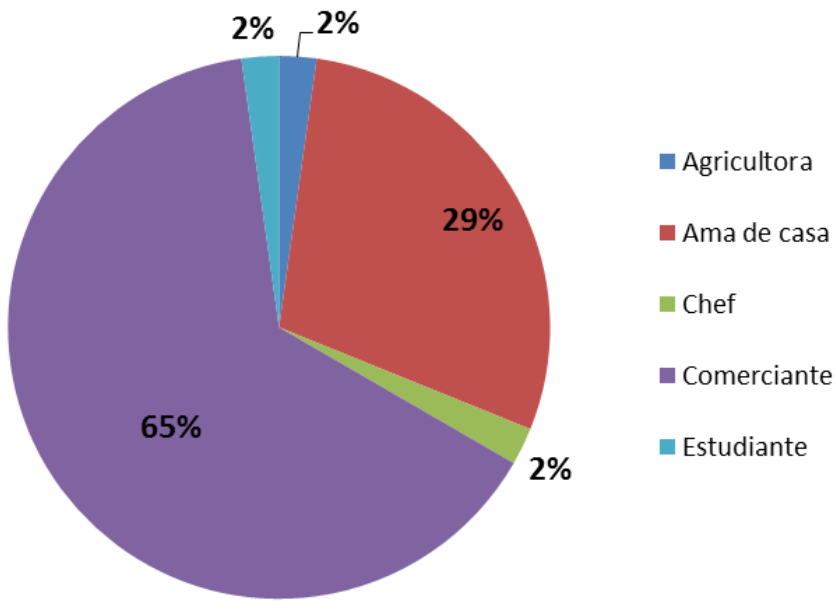

Figura 2: Ocupaciones de las personas entrevistadas en los mercados de Pichincha.

\section{Nomenclatura de las especies de patos comercializados y sus usos}

En total se identificaron tres especies de patos: Cairina moschata, Anas platyrhynchos domesticus Linnaeus, 1758 y Anser anser (Linnaeus, 1758) (Figura 4), cuya nomenclatura local se especifica en la Tabla 2.

C. moschata corresponde a una especie nativa de Ecuador, cuyo macho es fácilmente diferenciable de la hembra por la presencia de carnosidades o carúnculas en el rostro (Figura 4C). Esta especie además de dos nombres castellanos, tiene otro específico en idioma kichwa, con el uso del vocablo runa posiblemente derivado de su condición de especie nativa. A diferencia de las otras dos especies, el pato chino A. domesticus y el ganso A. anser que son especies introducidas. El ganso es la especie de mayor tamaño y volumen de carne. Si bien los entrevistados desconocen el origen geográfico de las tres especies, no presentan dificultades para diferenciar in situ a cualquiera de ellas.

En la figura 4D se evidencian las diferencias entre los polluelos de ambas especies. Los polluelos del pato criollo $C$. moschata poseen una coloración parda oscura en su plumaje inicial, mientras que los de pato chino A. domesticus tienen un plumaje completamente amarillo. Cabe destacar que la mayor cantidad de ventas por parte de los comerciantes se obtiene de la especie nativa (C. moschata), pues es un ave de buenas características reproductivas de acuerdo a los entrevistados. 


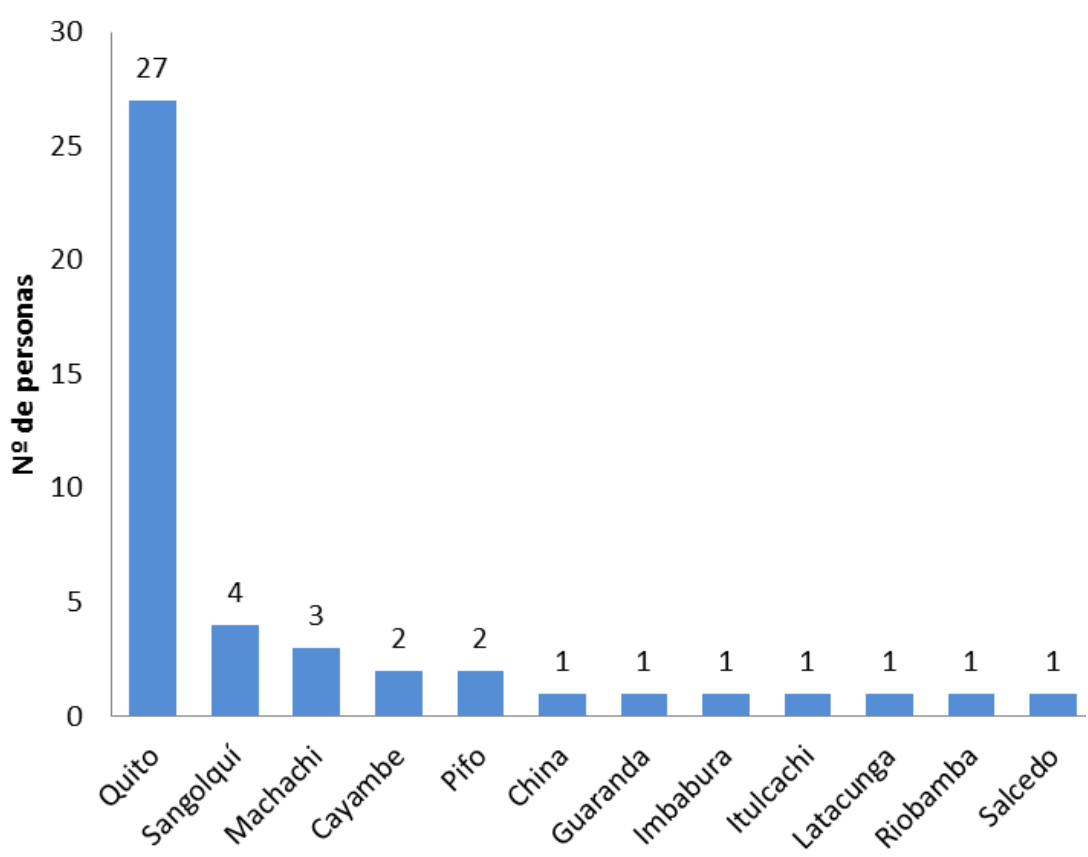

Figura 3: Lugar de origen de las personas participantes en las entrevistas de los mercados.

Tabla 2: Nomenclatura y categorías de uso para las especies de Anátidos.

\begin{tabular}{lll}
\hline Especie & Nombre común & Usos \\
\hline \multirow{4}{*}{ Anas platyrhynchos domesticus } & Pato chino & Alimentación \\
& Pato Pekín & Crianza \\
& Pato Blanco & Mascota \\
& & Medicinal \\
Anser anser & Ganso & Alimentación \\
& Pato Ganso & Crianza \\
& Pato criollo & Alimentación \\
Cairina moschata & Pato nacional & Crianza \\
& Pato Runa & Didáctico \\
& & Mascota \\
& & Medicinal \\
\hline
\end{tabular}

Para el caso del ganso A. anser se obtuvo un único registro en la feria de Machachi, y sus usos se limitan a la categoría de alimentación, con el consumo de las aves y sus huevos. Las categorías de uso son similares para las especies C. moschata y A. domesticus (Figura 5), a excepción de la categoría de uso didáctico definida para una de ellas. La categoría más utilizada para todas las especies corresponde al uso alimenticio. Si bien las diferencias son mínimas, es rescatable un mayor porcentaje de respuestas de los entrevistados al referir a $C$. moschata como mascota respecto a A. domesticus.

Uso alimenticio: De acuerdo con los entrevistados, las aves pueden ser preparadas en una variedad de platos, entre los cuales mencionan: caldo de pato, pato al jugo o guisado, seco de pato, pato horneado, pato frito y pato al hueco como una preparación típica del país. Además, A. domesticus es utilizado en una receta extranjera conocida como pato laqueado a la pekinesa de acuerdo a un entrevistado. En esta categoría también se incluye el consumo de subproductos de estas aves tales como sus huevos, que también son muy comercializados debido al mayor número de vitaminas y nutrientes que poseen a diferencia de los huevos de gallina, de acuerdo a la opinión de los entrevistados. 

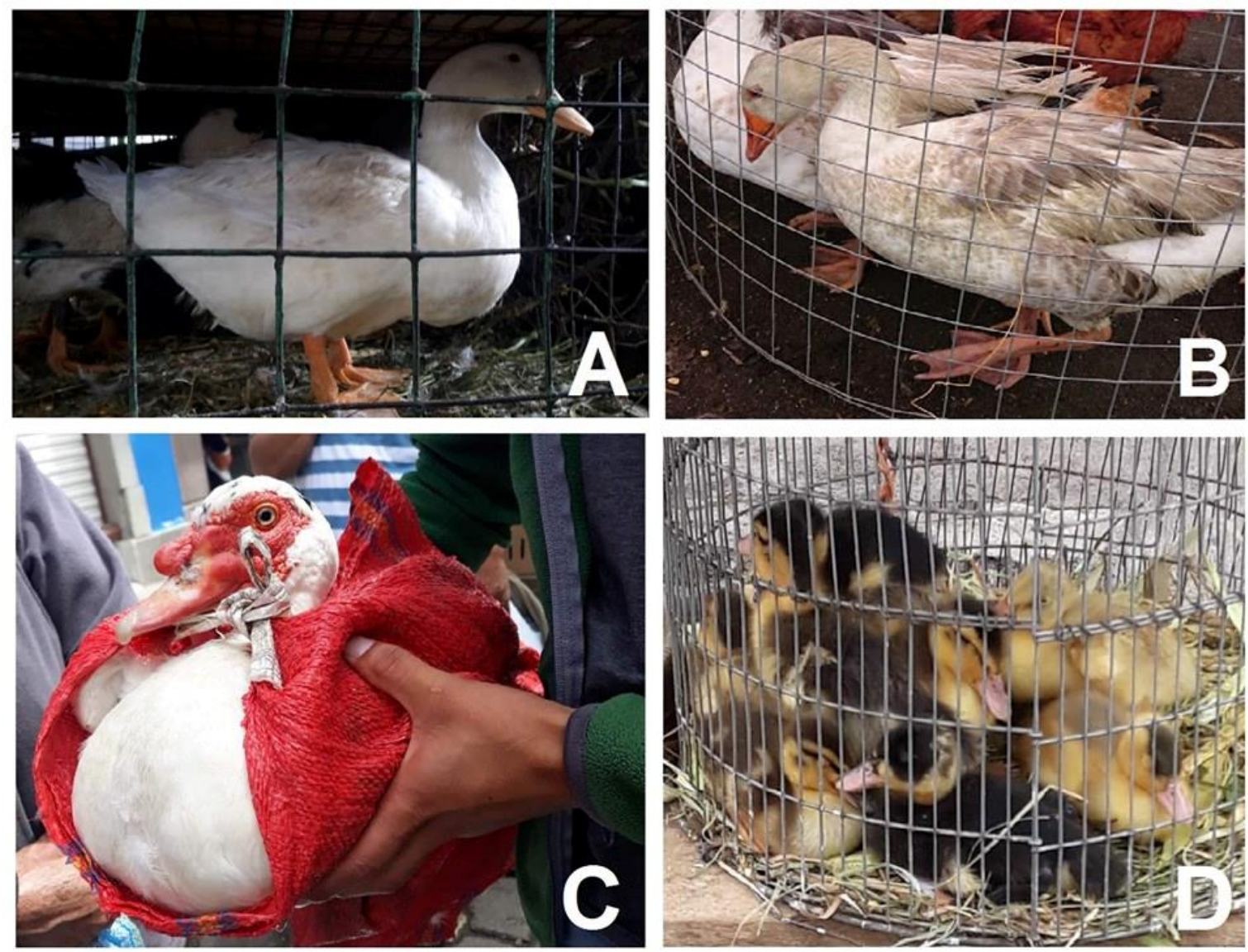

Figura 4: Ejemplares de patos en los mercados de Pichincha: (A) San Roque, A. domesticus. (B) Machachi, A. anser. (C) Sangolquí C. moschata. (D) Sangolquí, A. domesticus y C. moschata (polluelos).

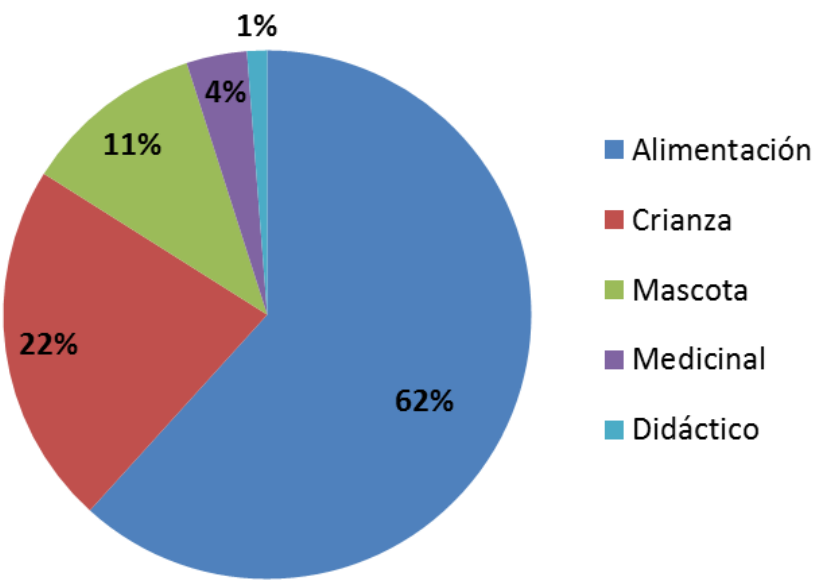

Figura 5: Categorías de uso registradas en los mercados de Pichincha para las aves pertenecientes a la Familia Anatidae.

Uso para crianza: Las personas entrevistadas revelaron que frecuentemente se compran los patos, en edad de polluelos o juveniles, para su crianza en el hogar, luego se los engorda para posteriormente utilizarlos como alimento o se busca su reproducción para la postura de huevos y crías destinadas al comercio local. Una de las entrevistadas mencionó que las hembras del pato criollo $C$. moschata tienen nidadas de 9 a 12 huevos y usualmente todos llegan a la eclosión bajo el cuidado materno hasta el día 12 de nacimiento de los polluelos. Por el contrario, se indicó que las hembras de A. domesticus y A. anser llegan a poner nidadas de tamaños similares, pero cuya eclosión es de apenas 2 a 4 huevos y los cuidados maternos son muy pocos o nulos, lo cual puede ser la principal causa de un menor éxito reproductivo respecto a la especie nativa. 
Uso medicinal: Dentro de este uso, los entrevistados reportaron principalmente el aprovechamiento de los huevos del pato criollo C. moschata para combatir los cólicos y malestares producto de la menopausia en mujeres. Además, también se reportó el uso de los huevos del pato chino A. domesticus para el dolor de garganta y la tos.

Uso de mascota: Las aves de acuerdo a los entrevistados tienen una utilidad como animales de compañía o mascotas, con único fin de tenerlos en exhibición como aves ornamentales debido a ciertas características físicas como el plumaje, el color y específicamente para C. moschata, la carúncula de gran tamaño. Los entrevistados también mencionaron otras características tales como su sociabilidad, inteligencia y el consumo de plantas consideradas como maleza para seleccionar a los patos como una mascota.

Uso didáctico: Este uso fue mencionado exclusivamente para el pato criollo C. moschata, y se relaciona con el empleo de esta especie en particular por parte de estudiantes con el fin de realizar disecciones o taxidermia.

\section{Comercio}

Entre los comerciantes entrevistados se obtuvo que un $72.5 \%$ de las aves en venta son compradas y un $27.5 \%$ son criadas por los vendedores. Los sitios de procedencia de las aves en su mayoría están ubicados por fuera de la provincia de Pichincha, incluyendo ciudades andinas como Ambato y Salcedo (Tabla 3) ubicadas en las provincias de Tungurahua y Cotopaxi, respectivamente. En el caso de los entrevistados de la feria de Sangolquí, se mencionó que las aves compradas en el sitio suelen ser revendidas en el mismo lugar.

Tabla 3: Lugar de procedencia de los patos comercializados en los mercados de Pichincha.

Lugar procedencia

\begin{tabular}{lc}
\hline Salcedo & 14 \\
Sangolquí & 13 \\
Latacunga & 9 \\
La Ofelia & 8 \\
Llano grande & 8 \\
Ambato & 6 \\
Quito & 3 \\
Saquisilí & 2 \\
Cayambe & 1 \\
Itulcachi & 1 \\
\hline
\end{tabular}

Los comerciantes indicaron que los animales procedían de distintas localidades, pudiendo ser adquiridos de acuerdo a las facilidades de cada uno. Se destaca como punto central de compra y venta al mercado de Sangolquí, esto posiblemente se deba a que en esta ciudad existe un día y lugar específico en el que tradicionalmente se venden únicamente aves de granja al público. Las comerciantes de los mercados de la Ofelia, Calderón y San Roque mencionaron que compran aves provenientes de dicho mercado para posteriormente criarlos y revenderlas en otras ferias de Quito. Además, tomando en cuenta que los días de feria son diferentes para cada mercado, suele ser el mismo comerciante quien vende sus patos en diferentes ferias mediante la modalidad de ventas rotativas.

Entre los compradores de aves entrevistados, se preguntó qué tan a menudo realizan esa actividad y se obtuvieron las siguientes cuatro categorías representadas en la Figura 6. Las personas que compraban de manera regular con una frecuencia semanal o mensual, generalmente lo hacían con fines económicos para su posterior comercio. Por el contrario, las personas que compran anualmente o en un lapso mayor de tiempo, lo hacen con la finalidad de criar las aves para la obtención de sus huevos y crías.

Las aves comercializadas han sido clasificadas en tres categorías de edad de acuerdo con los entrevistados cuyos porcentajes se especifican en la Figura 7. El estadio polluelo, corresponde a las crías aún con plumón y de coloración diferente a la de los adultos con edades mencionadas de 15 a 30 días de nacidos que son comercializados para su crianza. El estadio juvenil corresponde a aves de tres a cinco meses, como el grupo más comercializado ya que puede ser utilizado rápidamente como alimento o con fines reproductivos. Los entrevistados además mencionaron que el consumo del pato 
suele realizarse en esta etapa ya que su carne es más "tierna" o suave. Por último, la categoría de aves adultas corresponde a individuos de siete meses de nacidos en adelante.

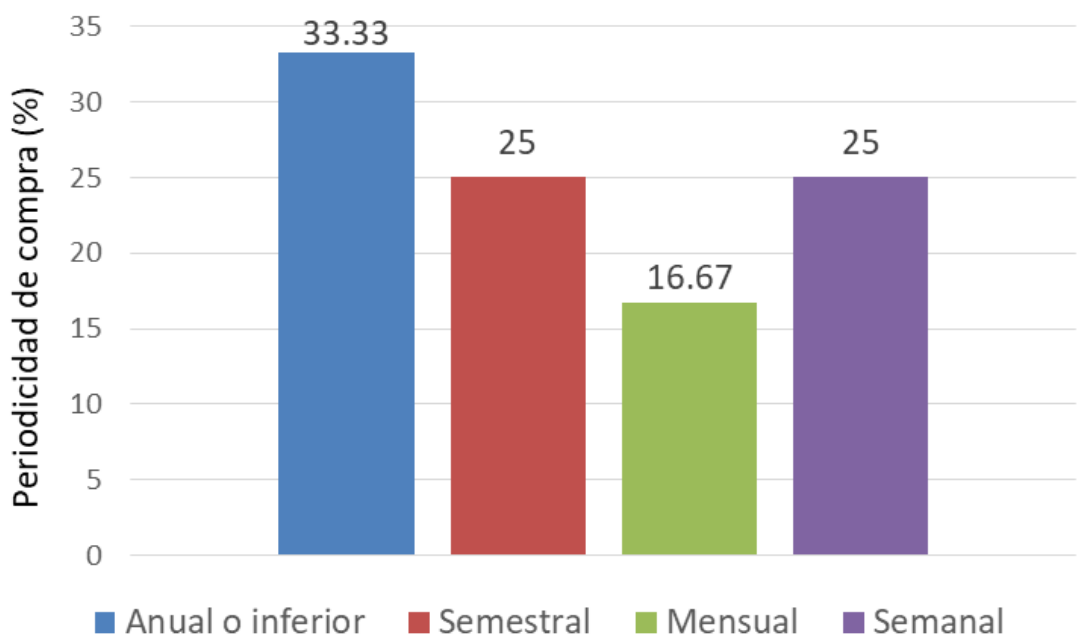

Figura 6: Periodicidad de compra de los patos por parte de personas asistentes a los mercados de Pichincha.

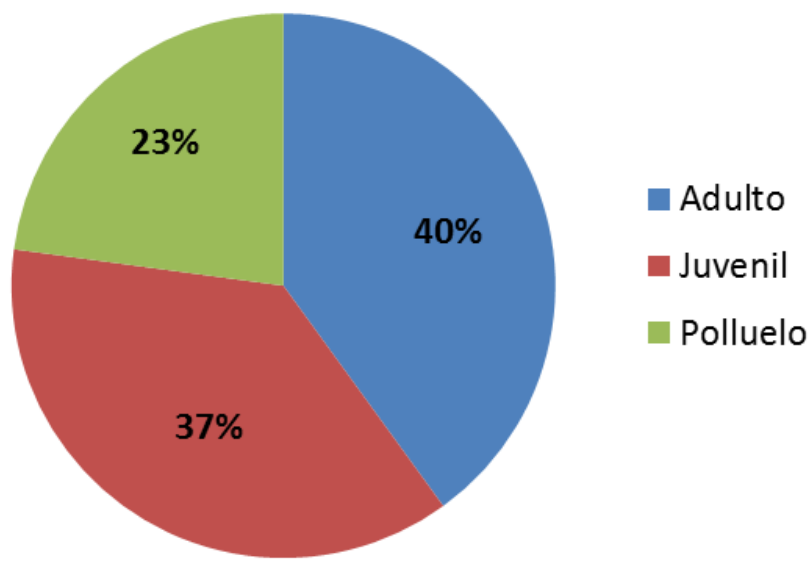

Figura 7: Estadio de las aves comercializadas.

\section{DISCUSIÓN}

Las aves tienen una alta representación en diversos aspectos para el ser humano, ya sean estos el alimenticio, medicinal, artesanal o simbólico, sin embargo, aún son escasos los estudios sobre sus usos y manejo a nivel de las comunidades (JAIMES et al., 2014). Históricamente la domesticación de los patos se ha visto favorecida por los beneficios que éstos traían a los humanos. Los patos fueron introducidos paulatinamente al entorno doméstico con el principal propósito de servir como controladores de insectos y guardianes del hogar, ya que emitían sonidos de alerta ante la presencia de extraños (ANGULO, 1998). Posteriormente, también fueron incorporados a la dieta humana como una fuente adicional de proteína.

Todos los procesos de domesticación tanto de C. moschata como de A. domesticus dieron paso a una serie de cambios en su fisiología tales como el aumento de masa corporal, un incremento en número de huevos, mayor desarrollo precoz y posibilidades de obtener crías durante todo el año, especialmente en la zona ecuatorial (FEDELLO, 1981; GUTIÉRREZ, 2013). Es probable que, debido a estos factores, aún se los mantenga en la esfera doméstica de Ecuador. En este estudio se encontró que el pato criollo es la especie más comercializada en los mercados visitados, con excepción del Mercado de San Roque, un importante centro de comercio de la capital, en donde hay una mayor venta del pato chino, una especie introducida. Esto se confirma con otro estudio en la región costera del país, en donde el pato criollo, como animal de corral, ha perdido terreno frente al pato chino desde que fue introducido desde Chile (TAMAYO, 2018). 
El pato chino, al presentar un desarrollo físico excepcional en sus primeros 50 días de vida, compite con el pato criollo en el aprovechamiento de su carne y grasa corporal (LÓPEZ et al., 2013; VELÁZQUEZ et al., 2016). Dada su buena resistencia a las condiciones ambientales y su rápido crecimiento, la cría de la especie introducida de pato se ha popularizado dentro de las comunidades rurales ecuatorianas en los últimos años, obligándolas a aprender y desarrollar nuevas técnicas para una adecuada nutrición que los haga aptos para la venta (MENDOZA, 2013; PAZ, 2013). Los resultados de este estudio reflejan una notable influencia de la especie exótica de pato en la demanda del mercado local, posiblemente generada por su crecimiento rápido y mayor rendimiento en carne y huevos.

Moura et al. (2018) mencionan que los patos de la familia Anatidae son preferidos por su gran masa corporal en contraste con otras aves silvestres comestibles; además, su carne es fina al paladar y nutritiva por lo que es apreciada a pesar de su alto contenido de grasa. La categoría de uso alimenticio representó en este estudio el mayor porcentaje, ya que los patos son un ingrediente importante en platillos nacionales tales como sopas o carnes, y en platos extranjeros. Naranjo (2011) destaca que varias especies de la familia Anatidae se preparan como una gallina casera en sudados después de despojarlos de plumas, cabeza y tripas, mientras que en la provincia de Tungurahua en Ecuador es conocida la técnica ancestral de pato al lodo (LLIGUISACA y ROHA, 2018).

En cuanto a los huevos, estos son consumidos principalmente en época seca, cuando abundan las puestas del pato criollo C. moschata. El pato chino A. domesticus también registró uso alimenticio principalmente por parte de cocineros de restaurantes chinos que lo aprovechan en sus menús para preparar platos típicos chinos como el pato laqueado a la pekinesa. El uso del pato chino evidencia la creciente afición por la cocina asiática en las zonas urbanas, debido a una variación de los hábitos alimenticios de las personas, con lo que se ha generado una mayor demanda hacia esta especie introducida (RUÍZ-FREIRE, 2018).

Para las comunidades latinoamericanas, los patos registran usos frecuentes como especies de caza de subsistencia, crianza de mascotas o como recursos medicinales o místicos locales (MARIZ et al., 2011). Por ejemplo, en las áreas rurales del municipio de Fagundes-Brasil, se han registrado especies de patos cuyo uso principal es el zooterapéutico para curar diferentes afecciones (ASEVEDO et al., 2011), de igual manera, las plumas son usadas para la producción de edredones, almohadas o vestimenta, al ser impermeables y aislantes (ALBUJA et al., 2012). En este estudio, no se encontró mayor influencia de la especie china en los demás usos reportados aparte del uso alimenticio, pues el pato criollo $C$. moschata tiene una mayor presencia en las comunidades rurales donde se lo encuentra como mascota y acompañante de las personas en su vida cotidiana. Sin embargo, la crianza de los patos siempre es menor a la crianza de otras aves de patio como las gallinas.

La avifauna introducida se ha convertido en una base socioeconómica importante en comunidades rurales. Sántiz Ruiz et al. (2014) destacan la crianza de gallinas debido a que requiere una mínima inversión y es capaz de generar rápidamente ganancias. Se han adaptado sistemas tradicionales para la crianza de estas especies, además, la infraestructura requerida es construida con materiales locales. Estos autores también mencionan que la crianza de especies de mayor tamaño requiere de suerte para obtener las condiciones óptimas. Está característica podría explicar el escaso comercio del ganso A. anser, la especie de mayor tamaño registrada en el estudio. Adicionalmente, estas aves son consideradas como reserva de dinero y solamente son comercializadas por requerimientos económicos urgentes o para el consumo en ocasiones festivas.

Si bien el consumo de la carne de pato no es muy popular frente a la de otras especies de aves de granja, esto se deba posiblemente al desconocimiento de su valor nutricional o a que es preferido como mascota (VELÁZQUEZ et al., 2018), es notable el uso de estas especies en la crianza a pequeña escala de acuerdo con los entrevistados, para aprovechar sus beneficios en el núcleo familiar o en el comercio local. A través de su comercio, las familias obtienen ingresos económicos adicionales con la venta de los ejemplares o de los huevos. Este sistema productivo aplicado a los patos es similar al encontrado para otras comunidades rurales, en donde son las mujeres principalmente quienes se encargan de la cría de las aves de granja para obtener carne y huevos, que luego son vendidos, o se usan para reproducción o autoconsumo (JIMÉNEZ et al., 2014), bajo un sistema de manejo de traspatio.

La avicultura de traspatio usa mano de obra familiar y genera bienes de consumo e ingresos que ayudan a solventar otras necesidades familiares (JUÁRES-CARATACHEA y ORTÍZ, 2001). A través de este sistema de manejo local, se contribuye a la conservación de especies nativas icónicas 
como es el caso del pato criollo $C$. moschata, cuya domesticación data de hace miles de años atrás en Ecuador. Posiblemente, las técnicas desarrolladas para esta especie por las mujeres locales podrían contribuir a la conservación de otras especies de patos silvestres, que actualmente registran amenazas por diversas actividades antrópicas en el país.

\section{CONCLUSIONES}

En los mercados estudiados en la provincia de Pichincha se reconocieron tres especies de patos de la familia Anatidae: una nativa, el pato criollo, nacional o runa Cairina moschata, y dos introducidas: el pato chino, Pekín o blanco Anas platyrhynchos domesticus, y el ganso Anser anser. Los usos entre las tres especies encontradas no difieren en gran medida, siendo la categoría de uso alimenticio la más relevante para las tres. Esta categoría incluye el consumo de su carne y huevos. Los entrevistados destacan una marcada preferencia por la especie nativa, al poseer mejores características reproductivas para su crianza, sin embargo, la creciente demanda de la especie introducida por restaurantes de comida china ha aumentado la crianza de esta especie de pato en el entorno rural.

Las personas vinculadas al comercio y consumo de las aves son mayoritariamente de sexo femenino y su edad promedio supera los 50 años. La ausencia de personas jóvenes vinculadas a la actividad puede ser un indicador de la pérdida del conocimiento local y los hábitos de consumo y uso de patos, especialmente de la especie nativa frente a otras especies de aves de granja

\section{AGRADECIMIENTOS}

A los comerciantes y compradores de los mercados visitados, quienes brindaron su conocimiento y colaboración en las entrevistas para la realización del presente trabajo realizadas con su consentimiento previo informado.

\section{REFERENCIAS BIBLIOGRÁFICAS}

ALBUJA, L; ALMENDÁRIZ, A; BARRIGA, R; MONTALVO, L; CÁCERES, F; ROMÁN, J.L. Fauna de Vertebrados del Ecuador. Quito, Ecuador: Instituto de Ciencias Biológicas-Escuela Politécnica Nacional, 2012. $490 \mathrm{p}$.

ANGULO, E. Interpretación Biológica acerca de la domesticación del pato criollo (Cairina moschata). Instituto de études andines, 1998. p. 19-31.

AVILEZ, J; CAMIRUAGA, M. Manual de crianza de patos. Temuco, Chile: Universidad Católica de Temuco, 2006. 84 p.

ASEVEDO, V, ALVES JA Y NÓBREGA RR. Utilização de aves silvestres por moradores do município de Fagundes, Semiárido paraibano: uma abordagem etno-ornitológica. Sitientibus série Ciências Biológicas, Feira de Santana, 11(2), p165-175, 2011.

BERNARD, H.R. Research Methods in Anthropology: Qualitative and Quantitative Approaches. 4 ed. New York: American Journal of Evaluation, 1996. 821 p.

CAZAMAJOR, P; MOYA, L. Quito, Aspectos geográficos de su Dinamismo: Los mercados y Ferias de Quito. Quito: Centro Ecuatoriano de Investigación Geográfica, 1984. 89 p.

CONSEJO PROVINCIAL DE PICHINCHA. Plan general de desarrollo provincial 2002-2022. Quito:

Prefectura de Pichincha, 2002. 367 p.

vaFEDELLO, C. Perspectivas de la cría de patos y ocas. Rivista di Avicoltura, 1981. p. 27-29.

FREILE, J; RESTALL, R. Birds of Ecuador. London: Bloomsbury Publishing Plc, 2018. 656 p.

GUTIÉRREZ-CANTUÑA, E.B; GUAINILLA-MALDONADO, W.R. Uso y Valor Económico de Naesiotus quitensis (Pfeiffer, 1848) (Mollusca: Bulimulidae) como alimento tradicional en la Parroquia urbana Sangolqui, Cantón Rumiñahui, Ecuador. Ethnoscientia, 3, p. 1-6, 2018. Disponible en: <http://www.ethnoscientia.com/index.php/revista/article/view/154>. Acceso en: 10 jun. 2019

GUTIÉRREZ, A. Dioses, símbolos y alimentación en los Andes. Interrelación hombre-fauna en el Ecuador prehispánico. Quito: Ediciones Abya Yala, 2009. 667 p.

GUTIÉRREZ, S. Efecto del manejo de patos mejorados bajo dos sistemas de crianza sobre los parámetros productivos. Universidad Nacional de Trujillo, 2013. p. 5-7.

JAIMES, M; GÓMEZ, G; PACHECO, N; REYES, S. Uso y manejo de la avifauna en San Miguel Tzinacapan, Municipio de Cuetzalan del Progreso, Pueblo, México. En: VÁSQUEZ-DÁVILA, M. Aves, personas y culturas Estudios de Etno-ornitología 1. Oaxaca: Red de Etnoecología y Patrimonio Biocultural, CONACYT, 2014. p. 243-259.

JIMÉNEZ-DÍAZ, J; VÁSQUEZ-DAVILA, M; NARANJO, E; JEREZ-SALAS, M. Las relaciones humanoaves en Lacanjá-Chansabay, Selva Lacandona, Chiapas, México. En: Vásquez-Dávila, M. 2014. Aves, personas y culturas Estudios de Etno-ornitología 1. Oaxaca: Red de Etnoecología y Patrimonio Biocultural, CONACYT, 2014. p. 83-106. 
JUÁREZ-CARATACHEA, A; ORTIZ, M. Estudio de la incubabilidad y crianza en aves criollas de traspatio. Revista Veterinaria México, México D.F., 32(1), p. 27-32, 2001. Disponible en: <http: //www.biblioteca.org.ar/libros/90255.pdf. >. Acceso en: 16 abr. 2020

LÓPEZ, M; ORTIZ, S; MIER, CE. Grasa corporal de Patos Muscovy Cairina moschata y Pekín Anas platyrhynchos como fuente de biodiesel. Rev. Colomb. Cienc. Anim, Sincelejo, 5(1), p. 58-70, 2013.

LLACSAHUACHE, N. Uso de acidificante en el engorde de patos (Cairina moschata). 2019. Tesis (Pregrado) - Facultad de Zootecnia, Universidad Nacional de Piura, Perú.

LLIGUiSACA, G; ROHA, B. Análisis gastronómico del pato al lodo de la ciudad de Píllaro en la provincia de Tungurahua. 2018. Tesis (Pregrado), Universidad de Guayaquil, Guayaquil.

MARIZ, DM; PEREIRA, HF; NÓBREGA, RR. Avifauna silvestre como recurso alimentar em áreas de semiárido no estado do Rio Grande do Norte, Brasil. Sitientibus série Ciências Biológicas, Feira de Santana, 11(2), p. 177-183, 2011.

MCMULLAN M; NAVARRETE, L. Fieldbook of the Birds of Ecuador including the Galápagos Islands and common mammals. 2 ed. Quito: Ratty ediciones, 2017. 228 p.

MENDOZA, JC. Niveles de Gandul (Cajanus cajan), en la alimentación de pato Pekines (Anas platyrhynchos), en el cantón Quevedo. 2013. Tesis (Pregrado Ingeniería Agropecuaria), Universidad Técnica Estatal de Quevedo, Quevedo.

MINISTERIO DE CULTURA Y PATRIMONIO. Patrimonio Alimentario, Pato. Quito, 2016. Disponible en: 〈http://patrimonioalimentario.culturaypatrimonio.gob.ec/wiki/index.php/Pato>. Acceso en: 29 jul. 2019

MOURA, V; KESLLEY, H; FARIAS, R; DUARTE, R. Conhecimento, uso alimentar e conservação da Avifauna Cinegética: Estudo de Caso no Município de Patos, Paraíba, Brasil. Interciencia, Caracas, 43(7), p. 491-497, 2018.

NARANJO, A. Conocimiento y Uso local Asociado a la Avifauna de los Humedales de Piñalito, Wisirare, Malvinas y Sabanales en Orocué, Casanare (Colombia). 2011. Tesis (Pregrado Ecología) Facultad de Estudios Ambientales y Rurales, Pontificia Universidad Javeriana, Bogotá.

NOBREGA-ALVES, R. Zooterapia: importancia, usos e implicaciones conservacionistas. En: COSTANETO, E; SANTOS-FITA, D; VARGAS-CLAVIJO, M. Manual de Etnozoología. Una guía teórico-práctica para investigar la interconexión del ser humano con los animales. Valencia: Tundra Ediciones, 2009. p. 165-175.

PAZ, PP. Niveles de promotor de crecimiento orgánico (Extracto de quillaja) en la cría y engorde de pato pekin (Anas platyrhynchos) en el cantón Valencia. 2013. Tesis (Pregrado Ingeniería Agropecuaria), Universidad Técnica Estatal de Quevedo, Quevedo.

PAZOS-BARRERA, J. El sabor de la memoria: Historia de la cocina quiteña. Quito-Ecuador: FONSAL (Fondo del Salvamento del Patrimonio Cultural de Quito), 2008. 40 p.

RUÍZ-FREIRE, MF. Utilización de aves de caza criadas en el Ecuador en cocina de vanguardia. 2018. Tesis (Pregrado Tecnología en Alimentos y Bebidas), Universidad de las Américas, Quito.

SÁNTIZ RUIZ G; PEREZGROVAS R; RODRÍGUEZ G; ZARAGOZA L. Importancia socioeconómica y cultural de las gallinas locales en una comunidad Tsotsil de Chiapas, México. En: VÁSQUEZ-DÁVILA, M. Aves, personas y culturas Estudios de Etno-ornitología 1. Oaxaca: Red de Etnoecología y Patrimonio Biocultural, CONACYT, 2014. p. 119-132.

SANTOS, D; COSTA NETO, E; CANO-CONTRERAS, E. El quehacer de la Etnozoología. En: COSTANETO, E; SANTOS-FITA, D; VARGAS-CLAVIJO, M. Manual de Etnozoología. Una guía teórico-práctica para investigar la interconexión del ser humano con los animales. Valencia: Tundra Ediciones, 2009. p. 23-44.

STAHL, PW; MUSE, MC; DELGADO-ESPINOZA, F. New evidence for pre-Columbian Muscovy Duck Cairina moschata from Ecuador. Ibis, 148(4), p. 657-663, 2006.

TAMAYO, VH. Saberes ancestrales de la etnia montubia del litoral ecuatoriano en la producción pecuaria de la parroquia La Unión cantón Babahoyo. 2018. Tesis (Ingeniería Agropecuaria) - Facultad de Ciencias Agropecuarias, Universidad Técnica de Babahoyo, Babahoyo.

TIGMASA-MENA, Y.L. Las relaciones interpersonales de los grupos étnicos: indígenas, afroecuatorianos y mestizos. 2018. Tesis (Licenciatura en Educación Básica) - Facultad de Ciencias Humanas y Educación, Universidad Técnica de Cotopaxi, Latacunga.

VARGAS-CLAVIJO, M. Patrimonio Zoocultural: el mundo animal en las expresiones tradicionales de los pueblos. En: COSTA-NETO, E; SANTOS-FITA, D; VARGAS-CLAVIJO, M. Manual de Etnozoología. Una guía teórico-práctica para investigar la interconexión del ser humano con los animales. Valencia: Tundra Ediciones, 2009. p. 118-144.

VÁSQUEZ, M. Los patos (Anatidae): Recurso zoogenético de los grupos étnicos del sureste de México. Actas Iberoamericanas de Conservación Animal, Madrid, 22(4), p. 313-315, 2014.

VELÁZQUEZ, J.R; ROCA, M; HERNÁNDEZ, A. Caracterización de los ácidos grasos de la carne de pato (Cairina moschata). Ciencia y Tecnología de Alimentos, La Habana, 26(2), p. 18-23, 2016.

VELÁZQUEZ, JR; ROCA, M; RODRÍGUEZ, JL. Carne de pato (Cairina moschata): algunas consideraciones para su uso en productos cárnicos. Ciencia y Tecnología de Alimentos, La Habana, 28(2): 6571, 2018. 